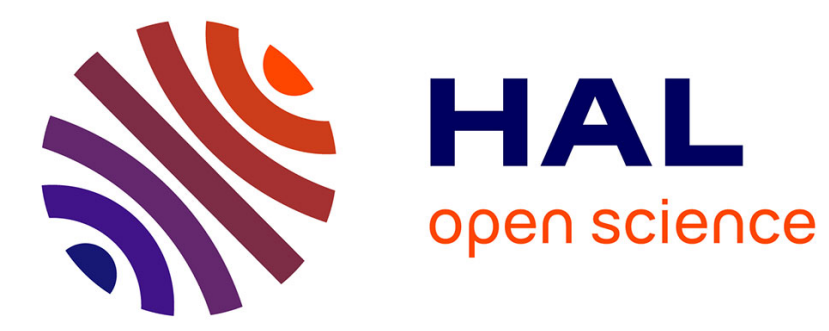

\title{
A Critique of the Adverse Childhood Experiences Framework in Epidemiology and Public Health: Uses and Misuses
}

\author{
Michelle Kelly-Irving, Cyrille Delpierre
}

\section{- To cite this version:}

Michelle Kelly-Irving, Cyrille Delpierre. A Critique of the Adverse Childhood Experiences Framework in Epidemiology and Public Health: Uses and Misuses. Social Policy and Society, In press, 10.1017/S1474746419000101 . hal-02088653

\section{HAL Id: hal-02088653}

\section{https://hal.science/hal-02088653}

Submitted on 3 Apr 2019

HAL is a multi-disciplinary open access archive for the deposit and dissemination of scientific research documents, whether they are published or not. The documents may come from teaching and research institutions in France or abroad, or from public or private research centers.
L'archive ouverte pluridisciplinaire HAL, est destinée au dépôt et à la diffusion de documents scientifiques de niveau recherche, publiés ou non, émanant des établissements d'enseignement et de recherche français ou étrangers, des laboratoires publics ou privés. 


\section{Social Policy and Society}

A Critique of the Adverse Childhood Experiences Framework in Epidemiology and Public Health: Uses and Misuses

\section{Michelle Kelly-Irving* and Cyrille Delpierre**}

*Equity research team, LEASP, Inserm, Iferiss, Université Toulouse III Paul Sabatier/, France E-mail: michelle.kelly@inserm.fr

${ }^{\star *}$ Equity research team, LEASP, Inserm, Iferiss, Université Toulouse III Paul Sabatier, France E-mail: cyrille.delpierre@inserm.fr

Adverse childhood experiences (ACEs) have emerged as a major research theme. They make reference to an array of potentially harmful exposures occurring from birth to 18 years of age and may be involved in the construction of health inequalities over the lifecourse. As with many simplified concepts, ACEs present limitations. They include diverse types of exposures, are often considered cumulatively, can be identified using prospective and retrospective approaches, and their multidimensional nature may lead to greater measurement error. From a public health perspective, ACEs are useful for describing the need to act upon complex social environments to prevent health inequalities at a population level. As the ACEs concept becomes popular in the context of policy interventions, concerns have emerged. As a probabilistic and population-level tool, they are not adapted to diagnose individual-level vulnerabilities, an approach which could ultimately exacerbate inequalities. Here, we present a critique of the ACEs framework, discussing its strengths and limits.

Keywords: Adverse childhood experiences; health inequalities, epidemiology, public health, policy. 


\section{Introduction}

The scientific literature on Adverse Childhood Experiences (ACEs) is burgeoning, and the term has also become commonplace outside the academic scientific milieu, including policy practice and social work. This article will examine the importance of the ACEs framework as it emerged in the field of epidemiology in the late 1990s and 2000s, and how it influenced research on the aetiology of health and the social determinants of health. We will also discuss the important societal issues that have emerged as the population-level epidemiological research has increasingly been used in other fields and at the individual level.

'Adverse childhood experiences' is a catch-all term that some authors have attempted to define and use more specifically (Brown et al., 2010). From a methodological perspective, the type of approaches mainly involve collecting recall data through questionnaires (Felitti et al., 1998), but some papers also identify ACEs using prospectively collected data (Kelly-Irving et al., 2013a). Across all fields of research recorded using the Web of Science, the number of papers referring to 'Adverse Childhood Experiences' in their title has increased from one publication in 1985 to two hundred and one in $2018^{1}$. The bulk of the increase in titles published on the topic occurred in 2010, and the biggest jump in numbers appeared between 2016 and 2017 (with an increase of 66 publications). This increase in the scientific interest in Adverse Childhood experiences, and use of the term in the titles of scientific publications is shown in Figure 1. 


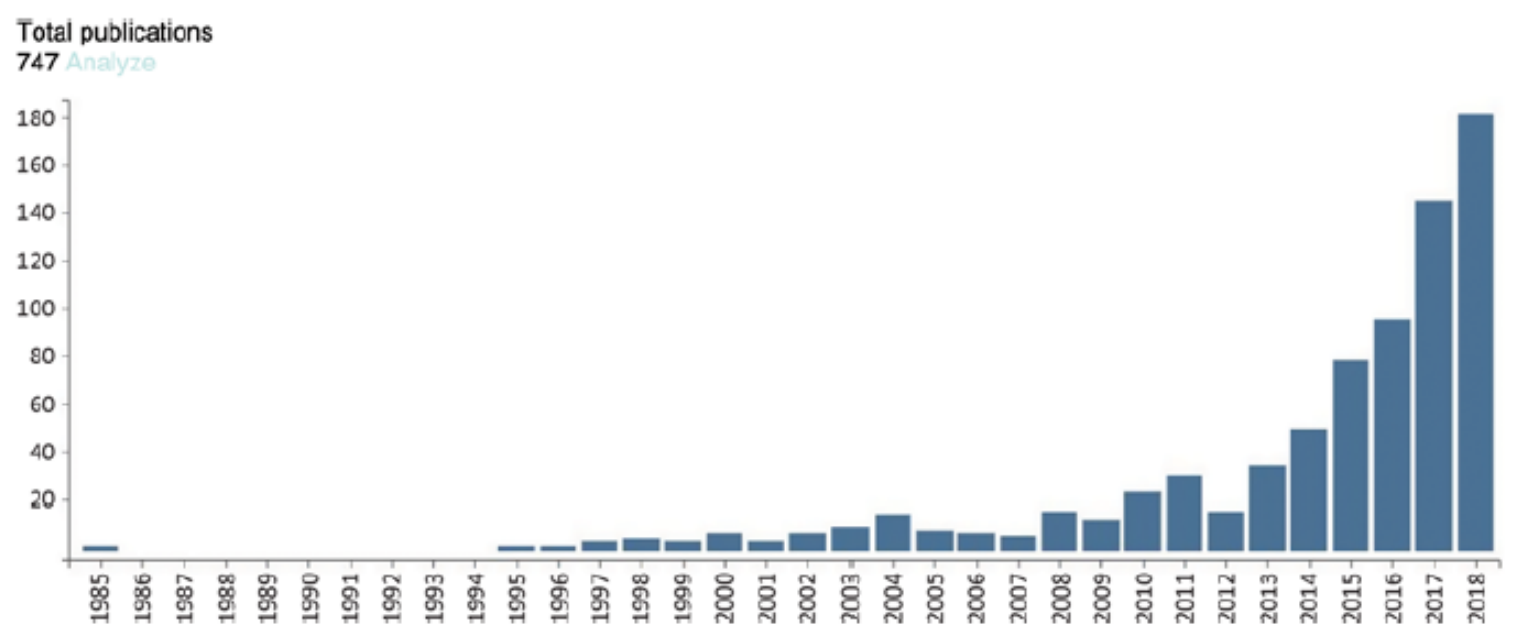

Figure 1. Number of scientific publications with 'Adverse childhood experience' in their titles per year recorded on the Web of science ${ }^{1}$

The increase is not only observed in the scientific field but also more general in prevention campaigns as shown (for example, see note 3). Indeed, a movement outside of science has also emerged advocating for awareness of Adverse Childhood Experiences, such as ACEs connection ${ }^{2}$. Such communities involve a wide array of interested parties, from survivors of trauma and abuse, to journalists, psychologists and child protection professionals.

In this article we will not attempt to summarise the entire scientific or non-scientific interest around ACEs. As epidemiologists and public health researchers, we will describe the influence of the ACEs framework as it emerged in the field of social epidemiology, and more specifically, regarding research into health inequalities. First, we will provide a brief outline of the field of social epidemiology and examine how ACEs research fit into the landscape. Second, we will outline the usefulness of evidence of ACEs, but also the limitations and problems that have emerged. Finally, we will discuss how research on ACEs in social epidemiology can move forward and how it may be interpreted more widely.

\section{Social epidemiology: from the black-box towards causal mechanisms}


Epidemiology is a quantitative discipline which acts essentially as a statistical tool-box to inform and provide evidence to the fields of medicine and public health. It has been criticised for its lack of theoretical framework and conceptual grounding (Krieger, 1994) relying above all on methodological principles. Susser and Susser traced epidemiological paradigms from the miasma theory of sanitary statistics, to the germ theory of infectious disease epidemiology, ending with the black-box theory of chronic disease epidemiology. The black-box 'related exposure to outcome without any necessary obligation to interpolate either intervening factors or even pathogenesis' (Susser and Susser, 1996: 671). The marginal field of social epidemiology also took the black-box approach for a long time. This involved describing the association between socioeconomic variables and health outcomes. In the 1980 s social medicine played a pivotal role in the British public arena, having a controversial impact upon the political agenda through the publication of the Black Report (Townsend and Davidson, 1982). The report documented and formally established that despite a growing economy and overall improvements in quality of life, health inequalities between the richest and poorest, across a gradient, had increased. The report's findings were suppressed by the Conservative government at the time (Townsend and Davidson, 1982: 3-11). The Black Report showed a class gradient for rates of long-standing illness throughout the 1970 s, with social class V, the most disadvantaged group, having the highest proportion of individuals reporting an illness limiting their everyday life (Townsend and Davidson, 1982).

The tradition of social medicine and social epidemiology in Britain, influenced by historical figures including Engels (Engels, 1987) largely focussed on the deleterious health effects of material deprivation and poverty. Namely, low income, poor housing and overcrowding were the main forms of social determinants examined in relation to health. In the early 2000s a debate played out in the academic literature between this 'neo-materialist' position (Lynch et al., 2000) and researchers defending the importance of psychosocial factors as determinants of health and health inequalities (Marmot and Wilkinson, 2001). The neomaterialist camp emphasised the importance of low incomes leading to deleterious material 
conditions for people, having to live in poorly maintained housing, not being able to afford adequate food and clothes, withe consequences on overall health. The psychosocial camp underlined the effects of social status, and status anxiety, where people with disadvantaged social status are exposed to chronic stress which has consequences on physiological and mental health. In itself, this debate was futile, since both pathways leading from social factors to health consequences are most likely operating together, and heavily intertwined. Yet, the debate was important in social epidemiology, shifting work on health inequalities from being a mere set of observations towards being an investigation of mechanisms. We moved beyond description, to asking how do social factors affect population health?

Desire to understand pathways and mechanisms was also pursued through research on what was then known as the Barker hypothesis or the foetal origins of adult disease. This work took an interest in understanding why socially deprived geographical areas appeared to have higher rates of cardiovascular disease 50 years later (Barker and Osmond, 1986). The research in this area offered an understanding of why genetic and adult risk factors did not provide sufficient explanation for cardiovascular disease incidence and mortality. Conceptual evolution in epidemiology has helped incorporate the foetal origins hypothesis into the more comprehensive and holistic conceptual framework that is the lifecourse approach (Kuh et al., 1997). In social epidemiology, the lifecourse framework emerged from the social sciences while also integrating concepts from the biological sciences. Understanding how health and disease are formed across the life span, and how the social environment is involved in this process has become a central question in epidemiology, and it is within this context that Adverse Childhood Experiences became a key area of research.

\section{Adverse Childhood Experiences emerge in social epidemiology}

In 1998, the 'ACEs study' described a strong graded relationship between a number of events and conditions in childhood deemed to be stressful which they named Adverse Childhood Experiences, and cause of death, (Felitti et al., 1998). The authors explained this association 
as an indirect relationship between the stressful conditions and mortality risk factors including health-related behaviours. Exposed individuals coped with adversity-induced stress by obtaining a pharmacological or psychological benefit from tobacco or alcohol use. The underlying thesis of this study was that 'stressful or traumatic childhood experiences have negative neurodevelopmental impacts that persist over the lifespan and that increase the risk of a variety of health and social problems'(Felitti et al., 1998). We will refer to this study as the 'point source' for the ensuing epidemiological interest in ACEs for pragmatic reasons, since this was the first study to use the term. However we fully recognise that previous work, especially by Michael Rutter (Rutter, 1980) on the subject of stressful conditions during childhood laid the ground for this subsequent literature. The ACEs study reported associations between ACEs and lung cancer (Brown et al., 2010), risk of suicide (Dube et al., 2001), depressive disorders (Chapman et al., 2004), ischaemic heart disease (Dong et al., 2004) and so on. Since then, many studies by other authors using different data, mainly from high income countries, have been conducted and published. Meta-analyses have now been conducted to examine the consistency of findings (Hughes et al., 2017; Holman et al., 2016). Hughes et al. (2017) conducted a meta-analysis of 37 studies measuring associations between multiple ACEs measured retrospectively and health outcomes. Their analysis supported substantially increased health risks to adults who reported multiple ACEs. Across all outcomes examined pooled odds ratio indicated increased risk of poor health among individuals with at least four ACEs compared with those reporting none. However, once outcomes are examined separately, a heterogeneity is observed between the effect sizes, with weak association for outcomes like physical activity and strong associations for outcomes such as poor mental health. Holman et al. (2016) undertook a meta-analysis to examine the potential association between ACEs and cancers. Across the 12 studies examined, ACE summary scores were associated with an increased risk of cancer in adulthood with all cancers pooled together. The studies which looked at cancer types separately, however, showed inconsistent findings, with only two out of the four showing any association between ACEs and the cancers. This is likely 
to reflect aetiological differences in specific cancer types. The authors' suggest that while early adversity may be associated with an increase in overall cancer incidence, the specific mechanisms by which effects occur may vary significantly according to the type of cancer.

Methodological issues arise with ACE studies, many of which have been discussed in the literature (for example, see Hartas, 2019). Across studies a heterogeneity exists in how ACEs were defined, for example, in some cases poverty and deprivation is included (Appleton et al., 2017). This may in some cases have its merits, however, it means that exposure to poverty and the material pathway between deprivation and health cannot be examine separately. One methodological flaw present in many ACE studies is the self-reported retrospective nature of the data. Usually adults are asked questions about trauma and adversities they may have experienced during childhood. Such questions are vulnerable to recall bias, where adults with poor health may be more likely to report adversity during childhood. Of course, this is often the only method available to researchers exploring the consequences of childhood adversity (Hardt and Rutter, 2004). However, some studies have since developed ACE measures using prospective data collected during childhood (Clark et al., 2010; Kelly-Irving et al., 2013a) or using a mixture of prospective and retrospective approaches (Houtepen et al., 2018). Houtepen et al. used a heterogeneous set of variable types to construct their ACEs. The majority of their early life data (0-8 years) was parent reported, but when the children were 8 years old they began self-reporting ACEs. Once in their twenties, the participants retrospectively reported on issues such as child maltreatment (several forms of abuse and neglect), violent behaviour of their own partner as well as whether their parents were violent towards each other. Overall, 89 per cent of all ACE variables were collected prospectively. This allowed the authors to take into account differences such as the sexual abuse rates prospectively reported by parents were much lower than those retrospectively self-reported by the participants. Prospectively collected information about ACEs presents a different set of methodological challenges, however. The data are collected by proxy, often from a parent or teacher who may not have full access to accurate information. 
In some cases it is possible to ask the children themselves, however it is probably inappropriate to question children directly about experiences of physical or sexual abuse. There is therefore a risk of misclassification bias, and under-reporting due to the sensitive nature of some issues or lack of awareness of any problems at the time.

The contribution of ACEs to understanding the construction of health inequalities is not apparent as a main objective of most studies, since in many cases the socioeconomic environment is not an object of explicit interest, but merely a background factor. For social epidemiologists, the body of work on ACEs and chronic pathologies is a convincing source of evidence for the psychosocial pathway between social exposures and health outcomes alluded to in the previous section. We suggest that the reason for this are fourfold. First, as we have mentioned, there was relative consistency in the findings across studies within the original ACE study and from other sources using different data from different population. Second, the association between ACE and health persists after adjustment for material deprivation or poverty suggesting that other mechanisms than those based on a 'neo-materialist' approach are at play. Third, social-to-biological plausibility was present, meaning that the theoretical links made between exposure to ACEs and biological processes appeared to make sense in the results (clustering of exposures, dose-response association with disease). Fourth, new developments in science on social embedding, embodiment or 'the social to biological transition' highlight how chronic stress may modify biological functioning. However, within the literature there is scope for considering ACEs within their socioeconomic context, and importantly, a need for a more detailed examination of mediating or indirect pathways between ACEs and health outcomes. ACEs are likely to occur along multiple causal pathways leading to a variety of adult health outcomes. For example, our own paper (Solis et al., 2015) highlighted that the relationship between ACEs and allostatic load at age 45 was mainly mediated through health behaviours, especially smoking, and wealth accumulation in adulthood. However, considerable further research into the mediation pathways between 
ACEs and health, especially on the mediating or moderating effects of social support or social capital, would provide an important contribution to this field.

Beyond merely trying to describe associations, the ACEs framework also attempts to understand how the relationships might work by referring to a literature on neurobiology, and notably the biology of stress (Shonkoff and Philips, 2000). The main argument and rationale explaining these outcome-wide associations was that living through ACEs is likely to induce the activation of physiological stress responses, which, when activated for long periods of time are harmful to human biological functioning. Human perceptions and emotions can lead to physiological stress responses in various biological systems (neurological, immune, hormonal). Psychosocial stress alters neuroendocrine hormone levels and down-regulates cellular immune responses mainly via glucocorticoid and adrenergic signalling pathways (Lupien et al., 2009). Stressful intra-familial conditions occurring between conception into adolescence causing this cascade of physiological responses may lead to an adaptive biological response during sensitive periods of development. This may alter an individual's biology in the long term in a way that makes them vulnerable to chronic conditions and pathologies over their lifecourse. Many studies, including birth cohort where ACE is prospectively measured, identify a dose-response association, where an increasing number of accumulated adversities is associated with a higher risk of morbidity (Dube et al., 2003; Danese et al., 2009; Clark et al., 2010; Solis et al., 2015). This type of relationship observed in cohort studies where the chronology of events between ACE and health is respected, is convincing to epidemiologists because it relates to criteria outlined by Bradford Hill (1965), for establishing a causal relationship (Hill, 1965).

One study was carried out post-mortem where epigenetic modifications linked to the stress response system in different brain areas were found to be present among individuals who had died from suicide compared to those who had succumbed to other causes of death (Poulter et al., 2008). Adding weight to the biological plausibility that ACEs in human children may result in negative health outcomes was evidence from animal models which was 
complimentary to the epidemiological literature. The early life stress experiments conducted on rats, where rat pups were separated from their mothers and subsequently reunited with them, have been linked to epigenetic alterations on gene receptors involved in the stress response system, functional changes in physiological systems, and behavioural changes in rat mothers (Liu et al., 1997). These changes in maternal biology and behaviour were also associated with differences in stress reactivity in their offspring (Francis et al., 1999). These biological studies of early life stress have been re-examined in mice, and some researchers have attempted to examine non-experimental evidence from humans which may point towards similar conclusions. Of course, huge scientific precautions are needed when examining this biological literature. First, no one is claiming that rodents and humans are equivalent; there are a great many differences between them. However, animal models have the advantage of demonstrating causal relationships between exposures and outcomes. Second, a population of suicide victims who were diagnosed with depression is not equivalent to people who may have been exposed to a number of the ACE types of stressors. The evidence from animal models, child neurobiology, and epigenetic epidemiology must simply be examined as distantly related, affording a fuzzy picture of a complex puzzle.

This biological plausibility of the ACEs framework, consistency of findings and doseresponse relationships all contribute to the evidence that psychosocial experiences occurring during the first two decades of life are likely to set certain groups of the population on chronic disease health trajectories. This does not exclude the existence of alternative pathways via deprivation or poverty, and furthermore, there is a lot of evidence that poverty and deprivation underlie the exposure of certain populations to these adversities (Metzler et al., 2017). Material hardship and low education are not only likely to be possible sources of physiological stress, but also indicate a lack of resources which may enable buffering against exposure to stressors. The sensitivity of human physiology during childhood accompanied by the physical and psychological ability to respond to environmental challenges is the underlying reason provided explaining why ACEs are associated with poor health later in life. However, one must not get 
carried away with how this is framed. While, on the one hand there is evidence for 'biological marks of stress' (Ridout et al., 2018), this is quite simply a sign of normal functioning. If we were able to examine all of our molecular and physiological structures, we would find biological marks for many other of our experiences, and some of these would probably affect our health.

\section{ACEs and the misuse of epidemiological results}

Altogether, epidemiological and animal studies in this area show that ACEs, through biological mechanisms involving stress response systems, are likely to act upon subsequent health and therefore represent a potential target for public health intervention. However this evidence is being used in a discourse around the ACEs framework that is possibly stigmatising and harmful. While the epidemiological research we described on ACEs may be useful evidence for population-level or structural policies, it is an insufficient and ill-adapted tool for implementation by social workers, medical practitioners, child protection workers, and likely to stigmatise families and children. Numerous examples of the concept of 'ACEs', becoming a buzzword in itself, being used for diagnostic purposes can be found. One such example is that of the National Health Service in Scotland, which has formed an 'ACEs Hub'3. As with many of these initiatives put in place in the health care sector, the intention is commendable: to take social factors into account when aiming to improve health. In this particular example, practitioners are encouraged to use an ACE questionnaire with patients, though a caveat is expressed about usage in schools, which they say could be stigmatising. An example outside of the health sector is an advocacy website 'ACEs too high'4, to promote awareness about ACEs. One page asks visitors if they have 'Got Your ACE Score?', and invites them to answer questions, and find out how many ACEs they have. The individualised use of the original ACEs questionnaire poses many potential ethical questions. What can an individual do with this information? Will they fear for their health, or even their lives even though the majority of exposed people will not develop any ACE-related problems? Indeed, by individualising the problem, it seems to take on a deterministic form, and puts the onus on individuals to act. 
These examples are rooted in the epidemiological research, however, they reveal a misunderstanding of the concept of risk. Let us look at briefly at our own previous paper on cancer more closely (Kelly-Irving et al., 2013b). We showed that women who experienced two or more ACEs were twice as likely to report having had cancer by the age of 50 , compared to women with no ACEs. When we look closely at the absolute figures we see that this result is due to 23 per cent of the 641 women in the high ACE group who had cancer, being compared to 9 per cent of the 2483 women in the zero ACEs group who had cancer. While the relative likelihood of developing cancer in the higher ACEs group is greater compared to women with no ACEs, it is important to note that the majority of women in the high ACE group, 77 per cent of them, did not develop cancer. This is nearly always the case in the ACEs epidemiological literature. We must remember that the evidence is about showing probabilities, and is not highlighting deterministic directly causal relationships. Although such results indicate that among a sample of people with ACEs we can expect a certain number of disease cases based on probabilities, we do not know which individuals in this population will develop the disease. At an individual level, having experienced ACEs does not mean that one is going to get cancer or die because of them. Furthermore, by grouping ACEs together in a cumulative score, assertions about individual risk also make the assumption that the specific patterning of ACEs and their consequences is the same for every individual. However, at an individual level the severity, timing, duration of stressful life events are likely to have different and heterogeneous consequences for health.

The original ACE study authors, if anything, made very prudent links between their findings and public health prevention strategies. The authors discussed primary, secondary and tertiary public health strategies without ever promoting the use of their measure for identifying people, but rather referred to the need for structural change, improved health visitation schemes, and better awareness about the impacts of stressful life conditions (Felitti et al., 1998). Since, in the last few years, the public health community has begun using the ACEs keyword in the primary prevention and health promotion approaches. An example of the 
misinterpretation may be found in a report by Public Health Wales (Wales, 2015). On page five, the relative risk of being adversely negatively affected by ACEs is explained at the individual level, instead of as a relative increase in risk between two population groups. In some cases agencies are screening for ACEs, often using the questionnaire which was designed for the initial epidemiological study. This rather concerning issue of screening is raised by Finkelhor (Finkelhor, 2018), who places the issue of screening back into its public health context. Fundamentally, he asks what exactly should be screened for, and can such screening tools be used in relation to clear effective interventions behind which adequate resources are placed? He recommends a much wider consideration of ACEs beyond their original items, and emphasises the need for primary prevention strategies.

As well as policy strategy and briefing reports, a number of videos have been made by national public health agencies. In the two examples we will refer to here, the audience for the videos appears to be the lay public, with an aim of increasing awareness of adverse childhood conditions. In both videos an individual approach is taken; a male narrator describes his life growing up in difficult socioeconomic and psychosocial circumstances. Once again, the focus is on individuals. However, the two examples are distinctive.

The illustrated video promoted by Public Health England and Wales ${ }^{5}$ lasting 5 min 43 seconds shows a cartoon boy narrator blocking his ears while sounds of arguing and breaking glass filter in the background. He says that the fighting and drinking in his home is going to lead to him having problems at school, with alcohol and with the police. He says that he is likely to die sooner than he should, and doctors have told him that things are 'changing inside him'... The video goes on to trace this boy's delinquent adolescence and his middle age as an overweight diabetic smoker who has 'never had a proper job' and hits his kids. The extremely negative focus of this video narrative on the life trajectory of one boy feeds into a set of stereotypes about social disadvantage and marginalisation. Attributing the experience of ACEs to this set of identifiers is scientifically wrong but could also be extremely stigmatising, 
especially if ACEs are being used by social workers or child protection agencies or even by individuals themselves to 'diagnose' or identify a risk.

The second video is by the Centre for Disease Control in the United States, which has funded much of the epidemiological research on ACEs in the USA ${ }^{6}$. This video also depicts a cartoon person, the narrator appears to be a young adult, and his focus is more on his neighbourhood and community. While he does detail a number of stereotyped identities such as the single mother working multiple jobs, the focus tends towards showing the negative effects of poverty, and positive effects of people in the community acting as mentors for children and adolescents. The narrator explains the positive effects of 'big brother' mentorship schemes and afterschool sports clubs on the lives of the children in the neighbourhood. He says 'when schools start taken responsibility for ACEs, everybody wins', and explains five strategies for preventing ACEs that can be carried out within communities: Strengthening economic support for families; Changing social norms; Quality child care and early life education; Enhancing parenting skills; Intervening to lessen harms and prevent future risk. The narrative of this video is still coming from an individual about his own experiences, however places him as part of a group by referring to his community. It puts the onus on structural change, involving schools and employment schemes to make positive change. The five strategies cited are extremely ambitious, but the overall picture painted is that of group effort and solidarity.

The contrast between these two examples highlights the difficulty and the danger of simplifying a message about complex social issues in a meaningful way for individuals. The second one is a more accurate reflection of how the evidence on ACEs could be explained, as well as choosing a positive and empowering message about population and communities, more akin with health promotion approaches.

\section{Conclusion}


We hypothesise that the observed increase in interest in ACEs in the scientific literature potentially comes from a number of sources: the initial novelty factor of the theme as opposed to previous social epidemiological research on poverty and deprivation; the catchy term; the fact that data on ACEs can be collected using relatively inexpensive retrospective questionnaires, making such studies relatively easy to do; the fact that the ACE literature is linked with the neurobiological and biological embedding literature, gives it biological plausibility despite the self-reported nature of the data; and the fact that there is a lay consciousness and even mythology about the long term harms of childhood trauma (orphaned children seem disproportionately represented in fictional literature). However, such a sudden increase in interest may have contributed to the decontextualisation of ACEs from the wider socioeconomic landscape and to a mismatch regarding links with policy.

The potential health consequences of ACEs is a research topic where the targeted level for intervention must be the structural social context in which children are exposed to ACEs and socioeconomic disadvantage. By examining this context, the focus is placed upon conditions which may be adverse for child well-being, and how these conditions come about. The focus should not be placed on the individual and his or her responsibility, this being all the more important when taking an interest in children. Nor should the evidence on ACEs be used to incriminate parents, but rather to reveal the conditions, particularly social conditions, in which parents and children live and how they cope. Developing studies on the biological impacts of ACEs therefore means seeking and providing evidence for population-based actions and avoiding the possible stigmatisation of families and children who lack the means to act. Given the strong impact agenda promoted by academic funders contributing to academic career promotion in some cases, the potential for academics to allow simplistic messages to emerge from their work is present. Underlying this may also be the erroneous assumption that policy makers or the public cannot understand subtle issues, which is likely to become a self-fulfilling prophecy. As social epidemiologists working on these issues, it is important for us to highlight the all too frequent excesses and temptations that consist in 
translating, in simplistic, erroneous, stigmatising and counterproductive ways, the research produced on ACEs relating to children and families.

\section{Notes}

1 Web of science all databases 1950-2018 « Adverse childhood experience » in title (searched 11/10/2018)

2 https://www.acesconnection.com/

3 NHS Scotland (2017) Adverse Childhood Experiences (ACEs) http://www.healthscotland.scot/population-groups/children/adverse-childhood-experiencesaces/overview-of-aces

4 Aces too high (2011) https://acestoohigh.com/

5 Public Health Network Cymru (2017), Adverse childhood experiences https://www.youtube.com/watch?v=YiMjTzCnbNQ

6 Centre for Disease Control (2018) We Can Prevent ACEs https://www.youtube.com/watch?v=8gm-INpzU4g

\section{References}

Appleton, A. A., Holdsworth, E., Ryan, M. and Tracy, M. (2017) 'Measuring childhood adversity in life course cardiovascular research: a systematic review', Psychosomatic Medicine, $79,4,434-40$.

Barker, D. J. P. and Osmond, C. (1986) 'Infant mortality, chidhood nutrition, and ischaemic heart disease in England and Wales', The Lancet, 1077-81.

Brown, D. W., Anda, R. F., Felitti, V. J., Edwards, V. J., Malarcher, A. M., Croft, J. B. and Giles, W. H. (2010) 'Adverse childhood experiences are associated with the risk of lung cancer: a prospective cohort study', BMC Public Health, 10. 
Chapman, D. P., Whitfield, C. L., Felitti, V. J., Dube, S. R., Edwards, V. J. and Anda, R. F. (2004) 'Adverse childhood experiences and the risk of depressive disorders in adulthood', Journal of Affective Disorders, 82, 2, 217-25.

Clark, C., Caldwell, T., Power, C. and Stansfeld, S. A. (2010) 'Does the influence of childhood adversity on psychopathology persist across the lifecourse? A 45-year prospective epidemiologic study', Annals of Epidemiology, 20, 5, 385-94.

Danese, A., Moffitt, T. E., Harrington, H., Milne, B. J., Polanczyk, G., Pariante, C. M., Poulton, R. and Caspi, A. (2009) 'Adverse childhood experiences and adult risk factors for agerelated disease depression, inflammation, and clustering of metabolic risk markers', Archives of Pediatrics and Adolescent Medicine, 163, 12, 1135-43.

Dong, M., Giles, W. H., Felitti, V. J., Dube, S. R., Williams, J. E., Chapman, D. P. and Anda, R. F. (2004) 'Insights into causal pathways for ischemic heart disease: adverse childhood experiences study', Circulation, 110, 13, 1761-6.

Dube, S. R., Anda, R. F., Felitti, V. J., Chapman, D. P., Williamson, D. F. and Giles, W. H. (2001) 'Childhood abuse, household dysfunction, and the risk of attempted suicide throughout the life span: findings from the Adverse Childhood Experiences Study', JAMA, 286, 24, 3089-96.

Dube, S. R., Felitti, V. J., Dong, M., Giles, W. H. and Anda, R. F. (2003) 'The impact of adverse childhood experiences on health problems: evidence from four birth cohorts dating back to 1900', Preventive Medicine, 37, 3, 268-77.

Engels, F. (1987) 'The condition of the working class in England', American Journal of Public Health, 93, 8, 171-84.

Felitti, V. J., Anda, R. F., Nordenberg, D., Williamson, D. F., Spitz, A. M., Edwards, V., Koss, M. P. and Marks, J. S. (1998) 'Relationship of childhood abuse and household dysfunction to many of the leading causes of death in adults. The Adverse Childhood Experiences (ACE) Study', American Journal of Preventive Medicine, 14, 4, 245-58. 
Finkelhor, D. (2018) 'Screening for adverse childhood experiences (ACEs): cautions and suggestions', Child Abuse and Neglect, 85, 174-9.

Francis, D., Diorio, J., Liu, D. and Meaney, M. J. (1999) 'Nongenomic transmission across generations of maternal behavior and stress responses in the rat', Science, 286,5442 , 1155-8.

Hardt, J. and Rutter, M. (2004) 'Validity of adult retrospective reports of adverse childhood experiences: review of the evidence', Journal of Child Psychology and Psychiatry and Allied Disciplines, 45, 2, 260-73.

Hartas, D. (2019) 'Assessing the foundational studies on adverse childhood experiences', Social Policy and Society, doi: [typesetter to insert doi].

Hill, A. B. (1965) 'The environment and disease: association or causation?', Proceedings of the Royal Society of Medicine, 58, 5, 295-300.

Holman, D. M., Ports, K. A., Buchanan, N. D., Hawkins, N. A., Merrick, M. T., Metzler, M. and Trivers, K. F. (2016) 'The association between adverse childhood experiences and risk of cancer in adulthood: a systematic review of the literature', Pediatrics, 138(Supplement 1), S81-91.

Houtepen, L., Heron, J., Suderman, M., Tilling, K. and Howe, L. (2018) 'Adverse childhood experiences in the children of the Avon Longitudinal Study of Parents and Children (ALSPAC) [version 1; referees: 1 approved]', Wellcome Open Research, 3, 106.

Hughes, K., Bellis, M. A., Hardcastle, K. A., Sethi, D., Butchart, A., Mikton, C., Jones, L. and Dunne, M. P. (2017) 'The effect of multiple adverse childhood experiences on health: a systematic review and meta-analysis', The Lancet Public Health, 2, 8, e356-66.

Kelly-Irving, M., Lepage, B., Dedieu, D., Bartley, M., Blane, D., Grosclaude, P., Lang, T. and Delpierre, C. (2013a) 'Adverse childhood experiences and premature all-cause mortality', European Journal of Epidemiology, 1-14.

Kelly-Irving, M., Lepage, B., Dedieu, D., Lacey, R., Cable, N., Bartley, M., Blane, D., Grosclaude, P., Lang, T. and Delpierre, C. (2013b) 'Childhood adversity as a risk for 
cancer: findings from the 1958 British birth cohort study', BMC Public Health, 13, 1, 767.

Krieger, N. (1994) 'Epidemiology and the web of causation: has anyone seen the spider?', Social Science and Medicine, 39, 7, 887-903.

Kuh, D., Power, C., Blane, D. and Bartley, M. (1997) Social pathways between childhood and adult health, in D. Kuh and Y. Ben-Shlomo (eds.), A Life Course Approach to Chronic Disease Epidemiology, Oxford: Oxford University Press.

Liu, D., Diorio, J., Tannenbaum, B., Caldji, C., Francis, D., Freedman, A., Sharma, S., Pearson, D., Plotsky, P. M. and Meaney, M. J. (1997) 'Maternal care, hippocampal glucocorticoid receptors, and hypothalamic-pituitary-adrenal responses to stress', Science, 277, 5332, $1659-62$.

Lupien, S. J., McEwen, B. S., Gunnar, M. R. and Heim, C. (2009) 'Effects of stress throughout the lifespan on the brain, behaviour and cognition', Nature Reviews. Neuroscience, 10, 6, 434-45.

Lynch, J. W., Davey Smith, G., Kaplan, G. A. and House, J. S. (2000) 'Income inequality and mortality: importance to health of individual income, psychosocial environment, or material conditions', British Medical Journal, 320, 1200-04.

Marmot, M. and Wilkinson, R. G. (2001) 'Psychosocial and material pathways in the relation between income and health: a response to Lynch et al', British Medical Journal, 322, 1233-36.

Metzler, M., Merrick, M. T., Klevens, J., Ports, K. A. and Ford, D. C. (2017) 'Adverse childhood experiences and life opportunities: shifting the narrative', Children and Youth Services Review, 72, 141-9.

Poulter, M. O., Du, L., Weaver, I. C. G., Palkovits, M., Faludi, G., Merali, Z., Szyf, M. and Anisman, H. (2008) 'GABAA receptor promoter hypermethylation in suicide brain: implications for the involvement of epigenetic processes', Biological Psychiatry, 64, 8, 645-52. 
Ridout, K. K., Khan, M. and Ridout, S. J. (2018) 'Adverse childhood experiences run deep: toxic early life stress, telomeres, and mitochondrial dna copy number, the biological markers of cumulative stress', Bioessays, 40, 9, e1800077.

Rutter, M. (1980) 'The Long-term effects of early experience', Developmental Medicine and Child Neurology, 22, 6, 800-15.

Shonkoff, J. P. and Philips, D. A. (2000) From neurons to neighborhoods: the science of early childhood development, Washington: National academy press.

Solis, C. B., Kelly-Irving, M., Fantin, R., Darnaudery, M., Torrisani, J., Lang, T. and Delpierre, C. (2015) 'Adverse childhood experiences and physiological wear- and-tear in midlife: findings from the 1958 British birth cohort', Proceedings of the National Academy of Sciences of the United States of America, 112, 7, E738-46.

Susser, M. and Susser, E. (1996) 'Choosing a future for epidemiology: I. Eras and paradigms', American Journal of Public Health, 86, 5, 668-73.

Townsend, P. and Davidson, N. (1982) Inequalities in Health: the Black Report, Middlesex: Penguin.

Wales, P.H. (2015) Welsh Adverse Childhood Experiences Study, Wales: Public Health Wales NHS Trust. 